\title{
Control Groups: Not Truly Baseline
}

\author{
Tsee Leng Choy ${ }^{1,2}$, John F. Connolly ${ }^{1,3,4}$ \\ ${ }^{1}$ McMaster Integrative Neuroscience Discovery and Study (MiNDS), McMaster University, Hamilton, Canada \\ ${ }^{2}$ School of Health Sciences, Universiti Sains Malaysia, Kubang Kerian, Malaysia \\ ${ }^{3}$ Department of Linguistics and Languages, McMaster University, Hamilton, Canada \\ ${ }^{4}$ Department of Medicine, Division of Physical Medicine and Rehabilitation, McMaster University, Hamilton, \\ Canada \\ Email: jconnol@mcmaster.ca
}

Received 7 June 2014; revised 5 July 2014; accepted 15 July 2014

Copyright (C) 2014 by authors and Scientific Research Publishing Inc.

This work is licensed under the Creative Commons Attribution International License (CC BY).

http://creativecommons.org/licenses/by/4.0/

(c) (i) Open Access

\begin{abstract}
Although routinely used, control group criteria vary considerably in research. Drawing from aspects in our normative study on musical emotion perception, we show how a group of participants who would normally be considered a control group in most studies, display clear event-related potential (ERP) differences when grouped by affective assessment scores that are rarely administered to presumed "control" group candidates. This preliminary finding demonstrates the importance of careful screening of control group membership. Failure in this process can produce misleading observations, undermining the validity of results.
\end{abstract}

\section{Keywords}

Event-Related Potentials, ERPs, Emotion, Music, Alexithymia, Depression, Sampling Bias

\section{Introduction}

The use of control groups and conditions as baseline measures is standard practice in scientific research, although definitions and criteria vary across experiments. Few have questioned the necessity of control measures and their potential influence on experimental results. Recently, more stringent determination of control conditions in psychological intervention and pharmacological research, particularly the elimination of placebo effects, were advocated [1]. In their survey of video game players $(\mathrm{N}=400)$, placebo effects (improved performance in cognitive assessments after viewing video games) were found even in the active control group (slow paced non-action games like Tetris) as well as the experimental group (fast paced action games like Unreal Tournament), highlighting the importance of matching participant expectations when designing experiments [1]. More significantly, their findings have highlighted the re-evaluation of control conditions alongside the experimental 
question; a recommendation having clear implications beyond intervention and pharmacological research.

Although control group variability was not our main research focus, preliminary results of our normative study on affective priming and music [2] demonstrated significant group differences in the electrical brain responses (ERPs) of participants $(\mathrm{N}=48)$ in music emotion perception. What was interesting was that these participants would normally be considered a control group in most studies and were a representative sample of a normative and typical undergraduate population. However, when this sample was partitioned into four groups (12 participants per group) based on emotional characteristics, it became clear that "normative" or "representative" labels were misleading. Similar to [1], our results highlighted the importance of determining the nature of controls in accordance to the research question. We suggest additional deliberation and assessments be considered, particularly for research in the emotion domain. Before presenting the preliminary results of our research, there are several factors requiring explanation.

\section{Consider Emotion}

Selecting a valid control group is central in emotion research, as individuals can be affected by primary (e.g. family history of depression) or secondary (e.g. post-traumatic stress disorder) dispositions prior to the experiment. Emotion, feeling, affect and mood should first be distinguished as these terms are often used interchangeably in the literature. Emotion is most commonly defined as the neurophysiological and motor-expressive changes regulated by the neural system in response to a specific perception, object or event [3]. Feeling is the subjective, cognitive-experiential domain of an emotion; affect is the overall state encompassing emotion and feeling, incorporating other evaluative and valenced (range of positive and negative emotional value) elements like memories or preference; and mood is a less intensive, sustained state that lacks focus or an object [4]. As Damasio observed [5], “Affect is more general and can designate the whole subject matter we are discussingemotion, mood, feeling. It is the thing you display (emote) or experience (feel) towards an object or situation, any day of your life whether you are moody or not.”

\section{Consider Consciousness}

Following the proper designations for emotion, it becomes apparent how consciousness or more specifically, awareness increases from emotion to feeling, testament to the intricate relationship between emotion and consciousness. Consider how consciousness is amplified in emotionally charged situations, how emotions are dampened in drowsiness [6], and how both exist on a continuum. The precedence of emotion over cognition [7], its more basic nature and regulation in the older, more primitive brain regions give rise to 'affective consciousness' [8]. Given how closely emotion and consciousness are intertwined, deficiencies in one domain would inevitably impinge on the other. In this respect, assessing not just depression (reflecting how emotional disruption impacts consciousness) but also alexithymia (reflecting how a lack of consciousness impacts emotion) become pertinent.

\section{Consider Alexithymia}

Depression is a condition marked by continual, recurrent, diminished function and low mood persisting for at least a fortnight [9]. As it influences our feelings and perception [10], depression is frequently controlled for in emotion research. In contrast, alexithymia is a relatively new personality construct or condition gaining attention only in the past four decades [11] due to its relationship to a number of medical problems [12] [13]. Alexithymic individuals have impaired emotional regulation, with difficulty distinguishing between internal sentiments and external sensations [14].

Alexithymia has been termed "blindfeel” because they process emotion without being fully aware of it [15]. Just as blindsight and conscious vision are both visual processes distinguished only by a difference in conscious awareness, alexithymia ("blindfeel”) and conscious emotion are both emotional processes that differ in their level of consciousness [16]. Alexithymia has also been likened to a series of disconnects in the transfer of emotional information, where the fundamental break seems to be where emotions interact with cognition to become feelings. Thus, alexithymia enables the examination of emotion in its purest form [17].

Although alexithymia and depression are often co-morbid, both are distinct conditions [18]. Anti-depressants and/or psychotherapy are considered most efficacious in treating depression [19]. However, self-reflection difficulties in alexithymic individuals renders psychotherapy ineffective [3] [6] and there are currently no demon- 
strated pharmacological interventions for alexithymia. Depression is more common in females [19], impairing cognitive and executive function [10]. However, alexithymia is more prevalent in males [20], with alexithymic individuals being typically unimpaired intellectually, often using their strong cognitive skills to compensate for their lack of emotional awareness [6].

Streaming our representative sample of individuals drawn from a normal population into four groups (control, alexithymic, depressed, depressed alexithymic) after the relevant assessments (Twenty-item Toronto Alexithymia Scale/TAS-20 [21]; Bermond-Vorst Alexithymia Questionnaire-B/BVAQ-B [22]; Beck Depression Inventory II/BDI-II [23] enabled observation of the individual and combined effects of depression and alexithymia on emotional processing as manifested by both behaviour and ERPs. These participants were free from neurological, psychiatric, language or learning issues, with normal to corrected normal vision and hearing.

\section{Alexithymia: Increased, Less Automatic and Most Compromised Processing}

Our participants listened to happy or sad music primes ( 1000 ms film music excerpt) preceding spoken word targets "happy" or "sad" (music-word paradigm) or music targets that were happy or sad (music-music paradigm). The task in both paradigms was to decide if the emotional valence of the primes and targets matched or not. Musical emotion perception was examined with ERPs [2]. Perceiving emotions in music is naturalistic and primarily why most people listen to music [24]. Music most often conveys meaning through emotion, making it a suitable platform to assess emotion.

As illustrated in Figure 1(a) and Figure 1(b), group ERP differences are apparent in both paradigms. The larger response amplitudes of the alexithymic group (red line) compared to other groups in both paradigms reflects additional processing effort and is suggestive of compromised emotional function [2]. Although alexithymic individuals can perform comparably with controls behaviourally [25], they typically overcompensate for their poor emotional regulation through augmented responses, to avoid ignoring potentially harmful or useful stimuli. This extra effort, manifested as larger response amplitudes for emotional categorization (N300), recognition (P300) and integration or meaning (N400) processes [2] results in a more controlled, less automatic processing of emotion compared to non-alexithymic individuals [26]. Additional support for this interpretation comes from research indicating that N400 responses are sensitive to uncertainty and/or indecision about the meaning of a stimulus [27].

Alexithymia group responses are particularly clear in the music-word paradigm (marked deviation of red line) in contrast to the similar performances of other groups, whose responses cluster together (Figure 1(a)). Conversely, group response differences in the music-music-paradigm was more subtle (Figure 1(b)), likely reflecting the more interpretive aspects of music evaluation than word processing. That is, music targets are more subjectively interpreted than word targets simply because it is far less likely to mistake the target word "happy" for the word "sad" than it is to interpret what others may consider a "happy" piece of music as sad. This group response distinction also indicates differences in paradigm automaticity [2]. When processing is contained within a domain (e.g. music-music paradigm), less effort is needed as the initial stimulus has primed the successive target. In contrast, processing between domains (e.g. music-word paradigm) requires additional processing besides priming, to switch from music to word domains, similar to switch costs in task switching [28]. Thus, it is not surprising to see the alexithymic group having more trouble with the less automatic music-word paradigm, which requires more effort to begin with.

\section{Alexithymia "Normalizes" Depressed Alexithymia Compared to Depressed Individuals}

The individual and combined effects of alexithymia and depression manifested in the ERP group waveforms and topographical maps reflect what might be considered a modulatory role of alexithymia. Given the general response attenuation in depression, it is not surprising to see the depressed group (blue line) having the smallest ERP response amplitudes for both paradigms (Figure 1(a) and Figure 1(b)). In contrast to behavioural findings showing alexithymic individuals being more depressed and less responsive than depressed individuals [29] [30], we found the ERPs of the depressed alexithymic group (green line) appeared more responsive and closer to the controls (black line) (Figure 1(a) and Figure 1(b)). This intermediacy is logical as the alexithymic (red line) and depressed (blue line) groups fall at opposite sides of the response spectrum. Hence, the simultaneous manifestations of alexithymia produces a more intermediate response as alexithymia "normalizes" depression in 


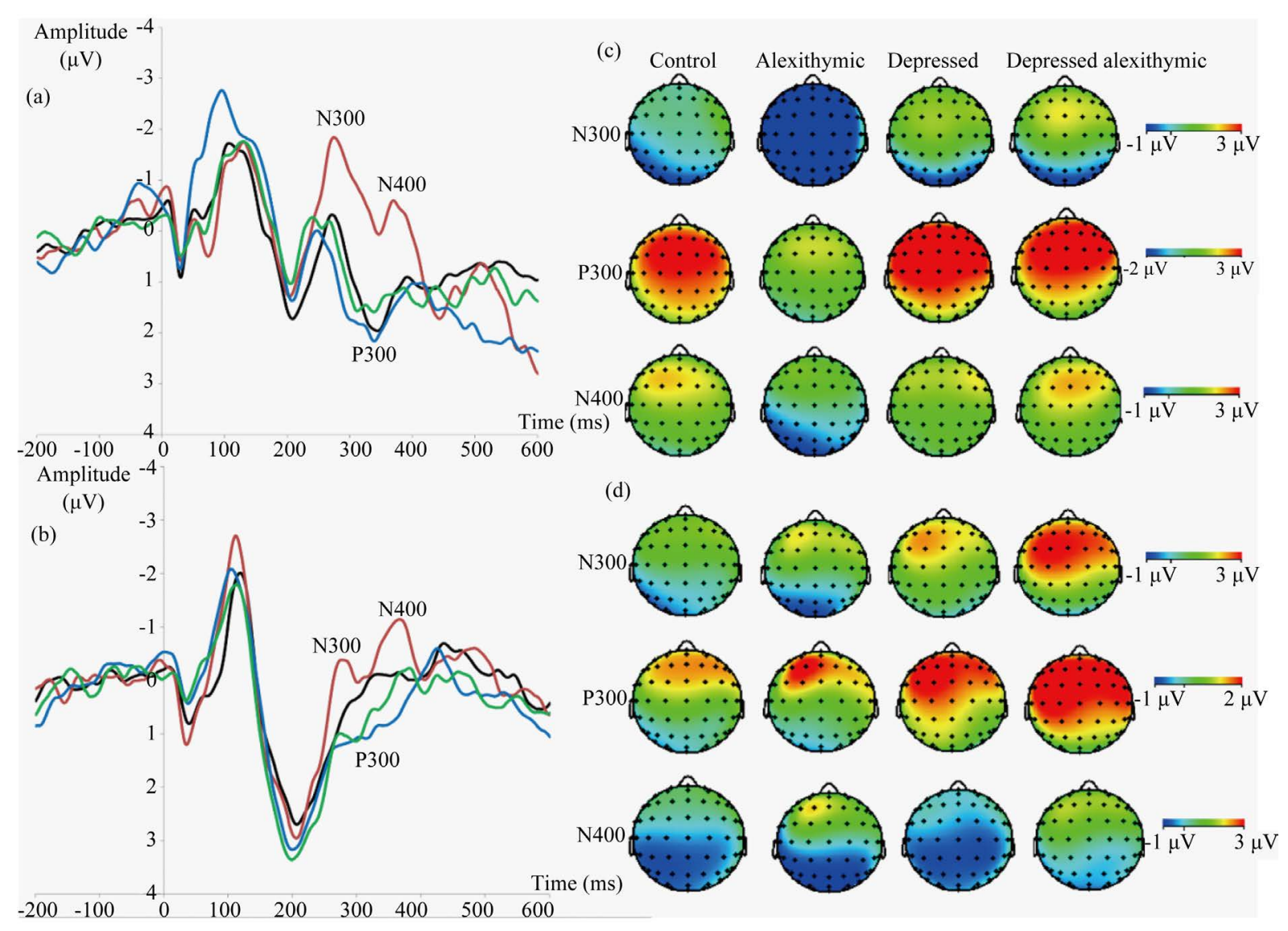

Figure 1. Participant N300, P300 and N400 ERP components reflecting stages of emotional processing grouped by participant group at electrode site $\mathrm{Pz}$, which best reflects experimental effects in all components for the normative database $(\mathrm{N}=$ 48). (a) Grand averages for the music-word paradigm. Lines denote groups: black (controls), red (alexithymics), blue (depressed), green (depressed alexithymics). (b) Similar ERP data for the music-music paradigm. (c) Topographical maps reflecting mean amplitudes across sites were effects were maximal for all components, for the music-word paradigm. Note the P300 scales differ from the other components and between paradigms to best showcase group differences. (d) Similar topographical data for the music-music paradigm. Negative is plotted up for ERPs, anterior is plotted up for topographical maps.

depressed alexithymia to some degree [2]. Our results also show ERPs to be a more sensitive and precise measure of alexithymia and depression as these group nuances were distinguished more clearly, unlike their behavioural counterparts. The topographical maps (Figure 1(c) and Figure 1(d)) also show the groups to display varying ERP component distributions.

\section{Future Directions}

Our four groups of participants were collectively meant to reflect a normative ERP database representing the general population. Nevertheless, the marked group ERP response differences have also prompted us to consider the variability of control groups as a whole. These significant group effect sizes are displayed in Table 1. A mixed $2 \times 2 \times 2 \times 4$ analysis of variance (ANOVA) with the repeated factors of target valence (happy, sad) and relatedness (related, unrelated) and a between groups factor (control, alexithymic, depressed, depressed alexithymic) was conducted. In the music-word paradigm, the P300 and N400 were statistically significant. For the P300, a main effect of group $(F(3,44)=10.42, p=0.0001)$, valence $(F(1,44)=10.23, p=0.0026)$, and a group $\mathrm{x}$ valence interaction $(F(3,44)=2.77, p=0.053)$ was found. These findings reflected valence affecting P300 responses differentially in the depressed and alexithymic groups, being particularly large for sad word targets in the depressed group (i.e. emotional negativity bias) and small for happy words targets in the alexithymic group. For the N400, a main effect of valence $(F(1,44)=6.79, p=0.013)$ reflected larger responses to happy targets compared to sad word targets for all groups, while a group trend $(F(3,44)=2.51, p=0.071)$ was attributed to 
Table 1. Effect sizes for group ERP differences for the music-word and music-music paradigms. Effect sizes (Adapted from Cohen 1988) are designated * for small $(0.15-0.40)$, ** for medium $(0.41-0.69)$ and *** for large $(0.70-\infty)$.

\begin{tabular}{clclc}
\hline \multirow{2}{*}{ ERP } & Music-word paradigm & Cohen's $\boldsymbol{d}$ & Music-music paradigm & Cohen's $\boldsymbol{d}$ \\
\hline \multirow{2}{*}{ N300 } & Control/alexithymic & $0.18^{*}$ & Control/alexithymic & $0.41^{* *}$ \\
& Control/depressed & -0.10 & Control/depressed & $-0.57^{* *}$ \\
& Control/depressed alexithymic & 0.03 & Control/depressed alexithymic & $-0.30^{*}$ \\
& Alexithymic/depressed & $-0.23^{*}$ & Alexithymic/depressed & $-0.86^{* * *}$ \\
& Alexithymic/depressed alexithymic & -0.14 & Alexithymic/depressed alexithymic & $-0.74^{* * *}$ \\
& Depressed/depressed alexithymic & 0.11 & Depressed/depressed alexithymic & $0.43^{* *}$ \\
P300 & Control/alexithymic & $0.19^{*}$ & Control/alexithymic & 0.07 \\
& Control/depressed & $-0.27^{*}$ & Control/depressed & $-0.17^{*}$ \\
& Control/depressed alexithymic & -0.05 & Control/depressed alexithymic & $-0.30^{*}$ \\
& Alexithymic/depressed & $-0.38^{*}$ & Alexithymic/depressed & $-0.22^{*}$ \\
& Alexithymic/depressed alexithymic & $-0.20^{*}$ & Alexithymic/depressed alexithymic & $-0.34^{*}$ \\
Depressed/depressed alexithymic & $0.19^{*}$ & Depressed/depressed alexithymic & $-0.15^{*}$ \\
Control/alexithymic & -0.07 & Control/alexithymic & 0.08 \\
& Control/depressed & $-0.24^{*}$ & Control/depressed & $-0.71^{* * *}$ \\
Control/depressed alexithymic & -0.02 & Control/depressed alexithymic & $-0.18^{*}$ \\
Alexithymic/depressed & $-0.15^{*}$ & Alexithymic/depressed & $-0.74^{* * *}$ \\
Alexithymic/depressed alexithymic & 0.05 & Alexithymic/depressed alexithymic & $-0.26^{*}$ \\
Depressed/depressed alexithymic & $0.19^{*}$ & Depressed/depressed alexithymic & $0.69^{* *}$ \\
\hline
\end{tabular}

larger N400 responses in the alexithymic group (i.e. additional processing efforts) compared to the depressed alexithymic group for all conditions. In the music-music paradigm, only the P300 was statistically significant. A main effect of group $(F(3,44)=13.21, p=0.0001)$ reflected larger responses in the depressed alexithymic compared to alexithymic group [2].

Alexithymia is neither typically controlled for nor routinely assessed in emotion research, and is likely to be a substantial confounding factor in present control groups, especially as alexithymia frequently overlaps with depression [18]. The individual and combined influence of alexithymia and depression are clearly manifested in both ERP waveforms (Figure 1(a) and Figure 1(b)) and topographical maps (Figure 1(c) and Figure 1(d)). Therefore, it is worthwhile for future research on emotion to incorporate the assessment of alexithymia separately and in combination with depression when determining control group criteria, to increase the validity of future results. Considering alexithymia has a prevalence rate between $10 \%-17 \%$ in healthy and $30 \%-60 \%$ in patient populations [31], accounting for its effects would have significant implications in both basic and clinical research in the emotion domain.

\section{Acknowledgements}

This research was supported by a doctoral scholarship from the Malaysian Ministry of Higher Education \& Universiti Sains Malaysia to Tsee Leng Choy and grants from the Canada Foundation for Innovation, the Ontario Research Fund, Natural Sciences and Engineering Research Council of Canada and McMaster University to John Connolly. The authors thank Magdalena Partyka \& Richard Mah for assistance with testing and figures.

\section{References}

[1] Boot, W.R., Simons, D.J. Stothart, C. and Stutts, C. (2013) The Pervasive Problem with Placebos in Psychology: Why Active Control Groups Are Not Sufficient to Rule out Placebo Effects. Perspectives on Psychological Science, 8, 445454. http://dx.doi.org/10.1177/1745691613491271

[2] Choy, T.L. (2013) Event-Related Potential (ERP) Responses to Music as a Measure of Emotion. Ph.D. Thesis, McMaster University, Hamilton. 
[3] Taylor, G.J., Bagby, M.R. and Parker, J.D.A. (1997) Disorders of Affect Regulation-Alexithymia in Medical and Psychiatry Illness. Cambridge University Press, Cambridge (UK). http://dx.doi.org/10.1017/CBO9780511526831

[4] Juslin, P.N. and Västfjäll, D. (2008) Emotional Responses to Music: The Need to Consider Underlying Mechanisms. Behavioural and Brain Sciences, 31, 559-621. http://dx.doi.org/10.1017/S0140525X08005293

[5] Damasio, A. (2000) The Feeling of What Happens-Body, Emotion and the Making of Consciousness. Cox \& Wyman Limited, Berkshire (Reading).

[6] Krystal, H. (1988) Integration and Self-Healing: Affect-Trauma-Alexithymia. The Analytic Press, Hillsdale, NJ.

[7] Dolan, R.J. (2002) Emotion, Cognition and Behaviour. Science, 298, 1191-1194. http://dx.doi.org/10.1126/science.1076358

[8] Panksepp, J. (2003) At the Interface of Affective, Behavioural and Cognitive Neurosciences: Decoding the Emotional Feelings of the Brain. Brain and Cognition, 52, 4-14. http://dx.doi.org/10.1016/S0278-2626(03)00003-4

[9] Thalpar, A., Colloshow, S., Pine, D.S. and Thalpar, A.K. (2012) Depression in Adolescence. Lancet, 379, $1056-1067$. http://dx.doi.org/10.1016/S0140-6736(11)60871-4

[10] Gotlib, I.H. and Joorman, J. (2010) Cognition and Depression: Current Status and Future Directions. Annual Review of Clinical Psychology, 6, 285-312. http://dx.doi.org/10.1146/annurev.clinpsy.121208.131305

[11] Taylor, G.J. and Bagby, M.R. (2004) New Trends in Alexithymia Research. Psychotherapy and Psychosomatics, 73, 68-77. http://dx.doi.org/10.1159/000075537

[12] Leweke, F., Leichsenring, F., Kruse, J. and Hermes, S. (2012) Is Alexithymia Associated with Specific Mental Disorders. Psychopathology, 45, 22-28. http://dx.doi.org/10.1159/000325170

[13] Lumley, M.A., Neely, L.C. and Burger, A.J. (2007) The Assessment of Alexithymia in Medical Settings: Implications for Understanding and Treating Health Problems. Journal of Personality Assessment, 89, 230-246. http://dx.doi.org/10.1080/00223890701629698

[14] Nemiah, J.C. and Sifneos, P.E. (1970) Psychosomatic Illness: A Problem in Communication. Psychotherapy and Psychosomatics, 18, 154-160. http://dx.doi.org/10.1159/000286074

[15] Lane, R.D., Ahern, G.L., Schwartz, G.E. and Kazniak, A.W. (1997) Is Alexithymia the Emotional Equivalent of Blindsight? Biological Psychiatry, 42, 834-844. http://dx.doi.org/10.1016/S0006-3223(97)00050-4

[16] Berridge, K.C. and Winkielman, P. (2003) What Is an Unconscious Emotion? The Case for Unconscious "Liking”. Cognition and Emotion, 17, 181-211. http://dx.doi.org/10.1080/02699930302289

[17] Frawley, W. and Smith, R.N. (2001) A Processing Theory of Alexithymia. Journal of Cognitive Systems Research, 2, 189-206. http://dx.doi.org/10.1016/S1389-0417(01)00029-8

[18] Honkalampi, K., Hintikka, J., Tanskanen, A., Lehtonen, J. and Viinamäki, H. (2000) Depression Is Strongly Associated with Alexithymia in the General Population. Journal of Psychosomatic Research, 48, 99-104. http://dx.doi.org/10.1016/S0022-3999(99)00083-5

[19] Kupfer, D.J., Frank, E. and Phillips, M.L. (2012) Major Depressive Disorder: New Clinical, Neurobiological and Treatment Perspectives. Lancet, 379, 1045-1055. http://dx.doi.org/10.1016/S0140-6736(11)60602-8

[20] Levant, R.F., Hall, R.H., Williams, C.M. and Hasan, N.T. (2009) Gender Differences in Alexithymia. Psychology of Men \& Masculinity, 10, 190-203. http://dx.doi.org/10.1037/a0015652

[21] Bagby, R.M., Parker, J.D.A and Taylor, G.J. (1994) The Twenty-Item Toronto Alexithymia Scale-I: Item Selection and Cross Validation of the Factor Structure. Journal of Psychosomatic Research, 38, 23-32. http://dx.doi.org/10.1016/0022-3999(94)90005-1

[22] Vorst, H.C.M. and Bermond, B. (2001) Validity and Reliability of the Bermond-Vorst Alexithymia Questionnaire. Personality and Individual Differences, 30, 413-434. http://dx.doi.org/10.1016/S0191-8869(00)00033-7

[23] Beck, A.T., Brown, G. and Steer, R.A. (1996) Beck Depression Inventory II Manual. The Psychological Corporation, San Antonio (Texas).

[24] Peretz, I. (1996) Can We Lose Memories for Music? The Case of Music Agnosia in a Nonmusician. Journal of Cognitive Neuroscience, 8, 372-380. http://dx.doi.org/10.1162/jocn.1996.8.6.481

[25] Franz, M., Schaefer, R., Schneider, C., Sitte, W. and Bachor, J. (2004) Visual Event-related Potentials in Subjects with Alexithymia: Modified Processing of Emotional Aversive Information? American Journal of Psychiatry, 161, 728-735. http://dx.doi.org/10.1176/appi.ajp.161.4.728

[26] Vermeulen, N., Luminet, O. and Corneille, O. (2006) Alexithymia and the Automatic Processing of Affective Information: Evidence from the Affective Priming Paradigm. Cognition and Emotion, 20, 64-91. http://dx.doi.org/10.1080/02699930500304654

[27] O’Rourke, T.B. and Holcomb, P.J. (2002) Electrophysiological Evidence for the Efficiency of Spoken Word Recogni- 
tion. Biological Psychology, 60, 121-150. http://dx.doi.org/10.1016/S0301-0511(02)00045-5

[28] Monsell, S. (2003) Task Switching. Trends in Cognitive Sciences, 7, 134-140. http://dx.doi.org/10.1016/S1364-6613(03)00028-7

[29] Vanheule, S., Desmet, M., Verhaeghe, P. and Bogaierts, S. (2007) Alexithymic Depression: Evidence for a Depression Subtype? Psychotherapy and Psychosomatics, 76, 315-316. http://dx.doi.org/10.1159/000104710

[30] Honkalampi, K., Saarinen, P., Hintikka, J., Virtanen, V. and Viinamäki, H. (2000) Factors Associated with Alexithymia in Patients Suffering from Depression. Psychotherapy and Psychosomatics, 68, 270-275. http://dx.doi.org/10.1159/000012343

[31] Parker, J.D.A., Keefer, K.V., Taylor, G.J. and Bagby, R.M. (2008) Latent Structure of the Alexithymia Structure: A Taxonomic Investigation. Psychological Assessment, 20, 385-396. http://dx.doi.org/10.1037/a0014262 
Scientific Research Publishing (SCIRP) is one of the largest Open Access journal publishers. It is currently publishing more than 200 open access, online, peer-reviewed journals covering a wide range of academic disciplines. SCIRP serves the worldwide academic communities and contributes to the progress and application of science with its publication.

Other selected journals from SCIRP are listed as below. Submit your manuscript to us via either submit@scirp.org or Online Submission Portal.
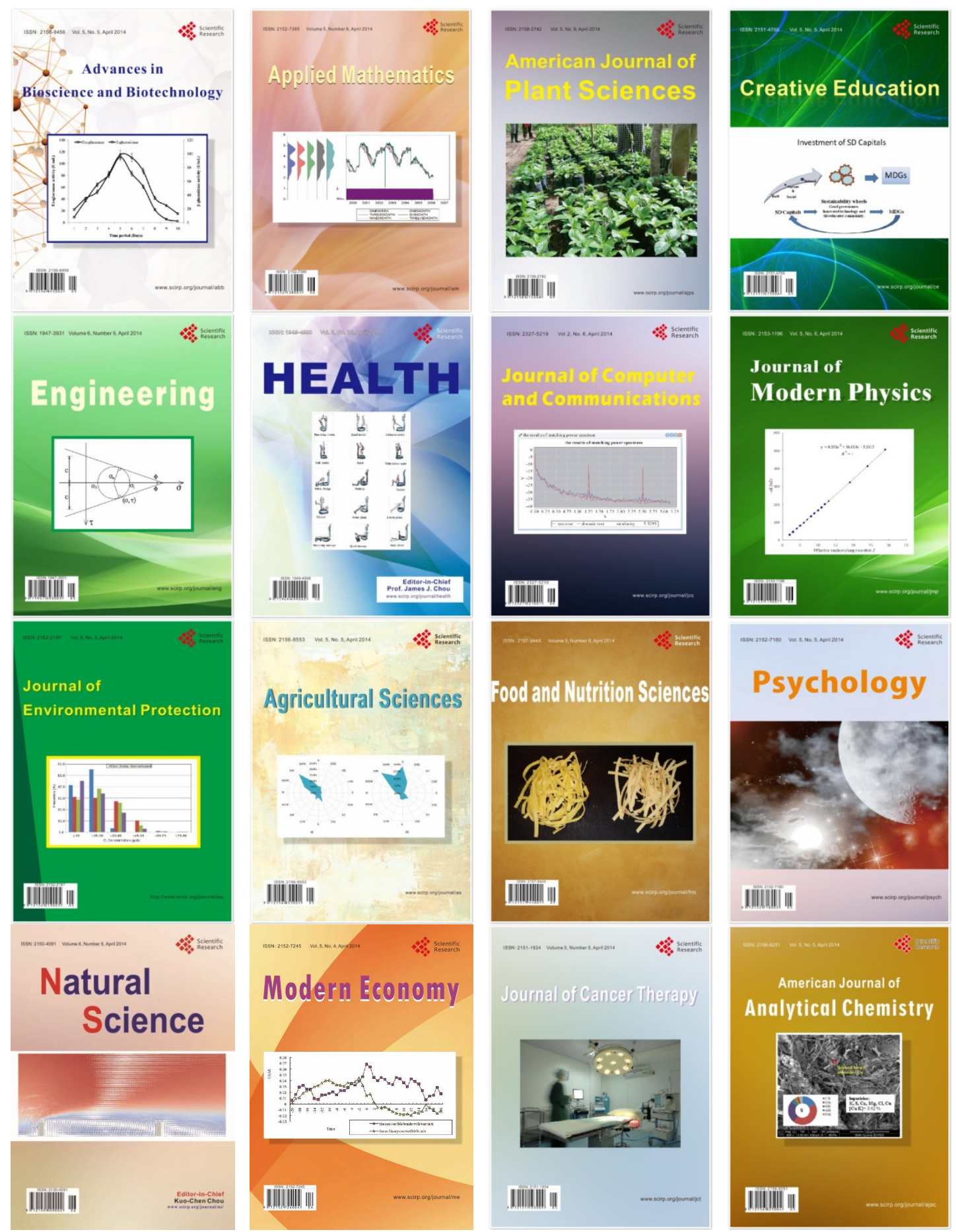